\title{
Retractorless translabyrinthine approach for resection of a large acoustic neuroma: operative video and technical nuances
}

\author{
JAMES K. LiU, M.D. ${ }^{1,2,3}$ AND Robert W. Jyung, M.D. ${ }^{2,3}$ \\ Departments of ${ }^{1}$ Neurological Surgery and ${ }^{2}$ Otolaryngology-Head and Neck Surgery, ${ }^{3}$ Center for Skull Base and \\ Pituitary Surgery, Neurological Institute of New Jersey, Rutgers University, New Jersey Medical School, Newark, \\ New Jersey
}

Large acoustic neuromas, greater than $3 \mathrm{~cm}$, can be technically challenging tumors to remove because of their intimate relationship with the brainstem and surrounding cranial nerves. Successful tumor resection involves functional preservation of the facial nerve and neurovascular structures. The translabyrinthine approach is useful for surgical resection of acoustic neuromas of various sizes in patients with poor preoperative hearing. The presigmoid surgical corridor allows direct exposure of the tumor in the cerebellopontine angle without any fixed cerebellar retraction. Early identification of the facial nerve at the fundus facilitates facial nerve preservation. Large acoustic tumors can be readily removed with a retractorless translabyrinthine approach using dynamic mobilization of the sigmoid sinus. In this operative video atlas report, the authors demonstrate their operative nuances for resection of a large acoustic neuroma via a translabyrinthine approach using a retractorless technique. Facial nerve preservation is achieved by maintaining a plane of dissection between the tumor capsule and the tumor arachnoid so that a layer of arachnoid protects the blood supply to the facial nerve. Multilayered closure is achieved with a fascial sling technique in which an autologous fascia lata graft is sutured to the dural defect to suspend the fat graft in the mastoidectomy defect. We describe the step-by-step technique and illustrate the operative nuances and surgical pearls to safely and efficiently perform the retractorless translabyrinthine approach, tumor resection, facial nerve preservation, and multilayered reconstruction of the skull base dural defect to prevent postoperative cerebrospinal fluid leakage.

The video can be found here: http://youtu.be/ros98Uxq VMw.

(http://thejns.org/doi/abs/10.3171/2014.V1.FOCUS13441)

KEY WoRDS $\quad$ acoustic neuroma $\quad \bullet \quad$ translabyrinthine approach $\quad \bullet \quad$ retractorless
technique $\quad$ video

Manuscript submitted September 30, 2013.

Accepted November 1, 2013.

Please include this information when citing this paper: DOI: 10.3171/2014.V1.FOCUS13441.

Address correspondence to: James K. Liu, M.D., Department of Neurological Surgery, Rutgers University, New Jersey Medical School, 90 Bergen Street, Suite 8100, Newark, New Jersey 07101. email: james.liu.md@rutgers.edu. 